\title{
RIEMANN'S METHOD AND THE PROBLEM OF CAUCHY. II. THE WAVE EQUATION IN $n$ DIMENSIONS ${ }^{1}$
}

\author{
J. B. DIAZ AND M. H. MARTIN
}

1. Introduction. In a recent paper ${ }^{2}$ Riemann's method for the solution of the problem of Cauchy for a linear hyperbolic partial differential equation $L(u)=0$ of second order for one unknown function $u$ of two independent variables $x, y$ was modified by the introduction of a line integral $I_{1}=\int\{B d x-A d y\}$ vanishing on closed paths. Here $A$ and $B$ are bilinear forms in the partial derivatives $u_{x}, u_{y}, v_{x}, v_{y}$; and $v$, the resolvent, is a properly chosen solution (analogous to Riemann's function) of an associate equation $M(v)=0$, the counterpart to the adjoint equation.

This modification opened the way to an extension of Riemann's method to the wave equation

$$
u_{x x}+u_{y y}-u_{t t}=0,
$$

in two dimensions. The line integral $I_{1}$ was replaced by an integral $I_{2}$ vanishing on closed surfaces and the associate equation $M(v)=0$ turned out to be the Euler-Poisson equation ${ }^{3}$

$$
M(v)=v_{\alpha \beta}+\frac{1 / 2}{\alpha-\beta}\left(v_{\alpha}-v_{\beta}\right)=0,
$$

with the resolvent

$$
v=\alpha+\beta+2[(\bar{t}-\alpha)(\bar{t}-\beta)]^{1 / 2}
$$

taking over the role of Riemann's function.

In the present paper the authors extend this method to the wave equation

$$
u_{x_{1} x_{1}}+\cdots+u_{x_{n} x_{n}}-u_{t t}=0
$$

in $n$ dimensions, $n \geqq 2$, with, as might be expected, an $n$-dimensional integral $I_{n}$, which vanishes over closed $n$-dimensional surfaces bounding $(n+1)$-dimensional volumes, replacing $I_{1}$ and $I_{2}$. The associate

Presented to the Society, December 27, 1951; received by the editors November 19, 1951.

1 This paper was sponsored by the Office of Naval Research.

2 M. H. Martin, Riemann's method and the problem of Cauchy, Bull. Amer. Math. Soc. vol. 57 (1951) pp. 238-249.

${ }^{8} \mathrm{G}$. Darboux, Lȩ̧ons sur la théorie générale des surfaces, $2 \mathrm{~d}$ ed., vol. II, p. 54 ff., Paris, 1914-1915. 
equation is now

$$
M(v)=v_{\alpha \beta}+\frac{(n-1) / 2}{\alpha-\beta}\left(v_{\alpha}-v_{\beta}\right)=0,
$$

and the resolvent is

$$
v=(\bar{t}-\alpha)^{(n-1) / 2}(\bar{t}-\beta)^{(n-1) / 2} .
$$

2. The Laplacian $\Delta_{2} u=u_{x_{1} x_{1}}+\cdots+u_{x_{n} x_{n}}$ in polar coordinates. Consider the generalization to $n$ dimensions of the well known space polar coordinate system $\phi, \theta, r$, in three dimensions, where

$$
\begin{array}{r}
x=r \cos \phi \sin \theta, \quad y=r \sin \phi \sin \theta, \quad z=r \cos \theta, \\
0 \leqq \phi<2 \pi, 0 \leqq \theta \leqq \pi, r \geqq 0,
\end{array}
$$

that is, coordinates $\phi, \theta_{1}, \cdots, \theta_{n-2}, r$ with

$$
\begin{array}{rlrl}
x_{1}=r \cos \phi \sin \theta_{1} \sin \theta_{2} \sin \theta_{3} \cdots \sin \theta_{n-2}, & 0 \leqq \phi<2 \pi, \\
x_{2}=r \sin \phi \sin \theta_{1} \sin \theta_{2} \sin \theta_{3} \cdots \sin \theta_{n-2}, & 0 \leqq \theta_{1} \leqq \pi, \\
x_{3}=r \cos \theta_{1} \sin \theta_{2} \sin \theta_{3} \cdots \sin \theta_{n-2}, & 0 \leqq \theta_{2} \leqq \pi, \\
x_{4}=r \cos \theta_{2} \sin \theta_{3} \cdots \sin \theta_{n-2}, & 0 \leqq \theta_{3} \leqq \pi, \\
\cdot \cdot \cdot \cdot \cdot \cdot \cdot \cdots \cdot \cdot \cdot, & & \cdot \\
x_{n-1}=r \cos \theta_{n-3} \sin \theta_{n-2}, & r \leqq \theta_{n-2} \leqq \pi, \\
x_{n}=r \cos \theta_{n-2}, & r \geqq 0 .
\end{array}
$$

The element of arc is given by

$$
\begin{aligned}
d s^{2}= & r^{2} \sin ^{2} \theta_{1} \cdots \sin ^{2} \theta_{n-2} d \phi \phi^{2}+r^{2} \sin ^{2} \theta_{2} \cdots \sin ^{2} \theta_{n-2} d \theta_{1}^{2}+\cdots \\
& +r^{2} d \theta_{n-2}^{2}+d r^{2}
\end{aligned}
$$

and if we write

$$
\begin{aligned}
y_{1} & =\phi, y_{2}=\theta_{1}, \cdots, y_{n-1}=\theta_{n-2}, y_{n}=r \\
g_{11} & =r^{2} \sin ^{2} \theta_{1} \cdots \sin ^{2} \theta_{n-2}, \\
g_{22} & =r^{2} \sin ^{2} \theta_{2} \cdots \sin ^{2} \theta_{n-2}, \cdots, g_{n-1, n-1}=r^{2}, \quad g_{n n}=1 .
\end{aligned}
$$

we shall have

$$
\Delta_{2} u=\frac{1}{g^{1 / 2}} \sum_{i=1}^{n} \frac{\partial}{\partial y_{i}}\left(\frac{g^{1 / 2}}{g_{i i}} u_{y_{i}}\right), g^{1 / 2}=r^{n-1} \sin \theta_{1} \sin ^{2} \theta_{2} \cdots \sin ^{n-2} \theta_{n-2} .
$$

If we set $f_{i-1}=g^{1 / 2} / r^{n-8} g_{i i}, i=1, \cdots, n-1$, so that 


$$
\begin{aligned}
& f_{0}=\csc \theta_{1} \sin \theta_{3} \sin ^{2} \theta_{4} \cdots \sin ^{n-4} \theta_{n-2} ; \\
& f_{1}=\sin \theta_{1} \sin \theta_{3} \sin ^{2} \theta_{4} \cdots \sin ^{n-4} \theta_{n-2} \text {; } \\
& f_{2}=\sin \theta_{1} \sin ^{2} \theta_{2} \sin \theta_{3} \sin ^{2} \theta_{4} \cdots \sin ^{n-4} \theta_{n-2} \text {; } \\
& f_{3}=\sin \theta_{1} \sin ^{2} \theta_{2} \sin ^{3} \theta_{3} \sin ^{2} \theta_{4} \sin ^{3} \theta_{5} \cdots \sin ^{n-4} \theta_{n-2} \text {; } \\
& f_{n-3}=\sin \theta_{1} \sin ^{2} \theta_{2} \cdots \sin ^{n-3} \theta_{n-3} \sin ^{n-4} \theta_{n-2} ; \\
& f_{n-2}=\sin \theta_{1} \sin ^{2} \theta_{2} \cdots \sin ^{n-2} \theta_{n-2} \text {; }
\end{aligned}
$$

we find

$$
\Delta_{2} u=f^{-1}\left[\frac{\partial}{\partial \phi}\left(\frac{f_{0}}{r^{2}} u_{\phi}\right)+\sum_{j=1}^{n-2} \frac{\partial}{\partial \theta_{j}}\left(\frac{f_{j}}{r^{2}} u_{\theta j}\right)\right]+u_{r r}+\frac{n-1}{r} u_{r},
$$

provided we write

$$
f=\sin \theta_{1} \sin ^{2} \theta_{2} \cdots \sin ^{n-2} \theta_{n-2}=f_{n-2} .
$$

We note in passing that the element of $(n-1)$-dimensional area on the unit sphere $r=1$ is

$$
d \omega_{n}=f d \phi d \theta_{1} \cdots d \theta_{n-2},
$$

and the $(n-1)$-dimensional area of the unit sphere is

$$
\omega_{n}=\int_{0}^{\pi} \cdots \int_{0}^{\pi} \int_{0}^{2 \pi} f d \phi d \theta_{1} \cdots d \theta_{n-2}=\frac{2 \pi^{n / 2}}{\Gamma(n / 2)} .
$$

3. A fundamental identity. Starting with the polar coordinates $\phi, \theta_{1}, \cdots, \theta_{n-2}, r$ in $n$-dimensional space we introduce coordinates $\alpha, \beta, \phi, \theta_{1}, \cdots, \theta_{n-2}$ in $(n+1)$-dimensional space-time by setting

$$
\alpha=t+r, \quad \beta=t-r,
$$

and term $\alpha, \beta$ characteristic coordinates, inasmuch as $\alpha=$ const., $\beta=$ const. are characteristic half-cones for the wave equation

$$
L(u)=\frac{f}{4}\left(u_{t t}-\Delta_{2} u\right)=0 .
$$

In these coordinates the operator $L(u)$ takes the form

$$
\begin{aligned}
L(u)= & {\left[u_{\alpha \beta}-\frac{(n-1) / 2}{\alpha-\beta}\left(u_{\alpha}-u_{\beta}\right)\right] f } \\
& -(\alpha-\beta)^{-2}\left[\frac{\partial}{\partial \phi}\left(f_{0} u_{\phi}\right)+\sum_{j=1}^{n-2} \frac{\partial}{\partial \theta_{j}}\left(f_{j} u_{\theta_{j}}\right)\right],
\end{aligned}
$$


with which we associate the operator

$$
M(v)=v_{\alpha \beta}+\frac{(n-1) / 2}{\alpha-\beta}\left(v_{\alpha}-v_{\beta}\right) .
$$

If we write

$$
\begin{aligned}
A & =f u_{\beta} v_{\beta}, & B & =-f u_{\alpha} v_{\alpha}, \\
\Phi & =f_{0} \frac{v_{\alpha}-v_{\beta}}{(\alpha-\beta)^{2}} u_{\phi}, & \Theta_{j} & =f_{j} \frac{v_{\alpha}-v_{\beta}}{(\alpha-\beta)^{2}} u_{\theta_{j}},
\end{aligned}
$$

$j=1, \cdots, n-2$, a simple calculation shows that (note that $v$ $=v(\alpha, \beta))$

(5) $A_{\alpha}+B_{\beta}+\Phi_{\phi}+\sum_{j=1}^{n-2} \frac{\partial \Theta_{j}}{\partial \theta_{j}}=\left(v_{\beta}-v_{\alpha}\right) L(u)+\left(u_{\beta}-u_{\alpha}\right) f M(v)$.

This identity plays the role of a Green's identity ${ }^{4}$ in our investigation, the part of the adjoint equation being taken over by the associate equation $M(v)=0$.

According to the generalized Green's theorem, the surface integral

$$
\begin{aligned}
I_{n}= & \int_{S_{n}}\left\{A d \beta d \phi d \theta_{1} \cdots d \theta_{n-2}+B d \alpha d \phi d \theta_{1} \cdots d \theta_{n-2}\right. \\
& \left.\quad+\Phi d \alpha d \beta d \theta_{1} \cdots d \theta_{n-2}+\cdots+\Theta_{n-2} d \alpha d \beta d \phi \cdots d \theta_{n-3}\right\} \\
= & \int_{S_{n}}\left\{A \nu_{\alpha}+B \nu_{\beta}+\Phi \nu_{\phi}+\cdots+\Theta_{n-2} \nu_{\theta_{n-2}}\right\} d S_{n}
\end{aligned}
$$

(where $\nu_{\alpha}, \nu_{\beta}, \cdots$ are the components of the unit outer normal to $S_{n}$ ) when extended around a closed $n$-dimensional surface $S_{n}$ bounding an $(n+1)$-dimensional volume $V_{n+1}$ can be expressed as a volume integral over $V_{n+1}$, namely

$$
\int_{V_{n+1}}\left(A_{\alpha}+B_{\beta}+\Phi_{\phi}+\sum_{j=1}^{n-2} \frac{\partial \Theta_{j}}{\partial \theta_{j}}\right) d \alpha d \beta d \phi d \theta_{1} \cdots d \theta_{n-2} .
$$

The following lemma is now obvious.

LEMma. The surface integral $I_{n}$, taken around a closed $n$-dimensional surface $S_{n}$, vanishes whenever $u$, $v$ are regular solutions of $L(u)=0$, and its associate equation $M(v)=0$, respectively.

"Compare the "formule fondamentale" in the terminology of J. Hadamard, Le problème de Cauchy et les équations aux derivées partielles linéaires hyperboliques, Paris, 1932, chapter II, esp. p. 83. 
It is worth while to note that each of $A, B, \Phi, \Theta_{1}, \cdots, \Theta_{n-2}$ is a bilinear form in the partial derivatives of first order of $u$ and $v$ with respect to $\alpha, \beta, \phi, \theta_{1}, \cdots, \theta_{n-2}$.

4. The problem of Cauchy. As Cauchy data on the hyperplane $t=0$ in $(n+1)$-dimensional space-time we take

$$
\begin{aligned}
u\left(x_{1}, \cdots, x_{n}, 0\right) & =u^{0}\left(x_{1}, \cdots, x_{n}\right), \\
u_{t}\left(x_{1}, \cdots, x_{n}, 0\right) & =u^{1}\left(x_{1}, \cdots, x_{n}\right),
\end{aligned}
$$

the functions $u^{0}, u^{1}$ being given in advance. Let $P_{\boldsymbol{l}}$ denote the point with coordinates $\left(\bar{x}_{1}, \cdots, \bar{x}_{n}, \bar{t}\right)$ in space-time. The solution of the problem of Cauchy requires the value $u\left(P_{l}\right)$ of the solution $u$ of $L(u)=0$ to be expressed in terms of the initial data $u^{0}, u^{1}$ carried by the part of the initial hyperplane $t=0$ contained within the ("retrograde") characteristic half-cone with vertex at $P_{\bar{t}}$, i.e., in terms of the initial data assigned to the points

$$
\left(x_{1}-\bar{x}_{1}\right)^{2}+\cdots+\left(x_{n}-\bar{x}_{n}\right)^{2} \leqq \bar{t}^{2}, \quad t=0 .
$$

We assume $\bar{t}>0$ and consider the $(n+1)$-dimensional conical volume $C$ bounded in space-time by the characteristic hypercone with vertex at $P_{\bar{t}}$ and the initial hyperplane $t=0$. The axis of $C$ is the straight line $P_{0} P_{l}$ in space-time traced out by $P_{t}$ as $t$ ranges from 0 to $\bar{t}$. If at each point $P_{t}$ we introduce polar coordinates $\phi, \theta_{1}, \cdots, \theta_{n-2}, r$ with pole at $P_{t}$, the conical volume $C$ is described by the inequalities

$$
\begin{array}{r}
C: \quad 0 \leqq \phi<2 \pi, \quad 0 \leqq \theta_{j} \leqq \pi, \quad 0 \leqq r \leqq \bar{t}-t, \quad 0 \leqq t \leqq \bar{t} \\
(j=1, \cdots, n-2) .
\end{array}
$$

When we take $\alpha, \beta, \phi, \theta_{1}, \cdots, \theta_{n-2}$ as rectangular coordinates in a second $(n+1)$-dimensional space, $C$ appears as a "wedge"

$W: \quad 0 \leqq \alpha \leqq \bar{t}, \quad-\alpha \leqq \beta \leqq+\alpha, \quad 0 \leqq \phi<2 \pi, \quad 0 \leqq \theta_{i} \leqq \pi$

$$
(j=1, \cdots, n-2) \text {. }
$$

That part of the boundary of $C$ formed by the mantle of the characteristic hypercone becomes the face $\alpha=\bar{t}$ of $W$; the base $t=0$ of $C$ is represented by the face $\beta=-\alpha$ of $W$; and the axis $P_{0} P_{l}$ of $C$ by the face $\beta=\alpha$ of $W$. The vertex $P_{l}$ of $C$ appears as the edge $\alpha=\beta=\bar{t}$ of $W$; the periphery of the base of $C$ (the intersection of the initial plane with the characteristic hypercone) is replaced by the edge $\alpha=-\beta=\bar{t}$ of $W$; and center $P_{0}$ of the base of $C$ by the edge $\alpha=\beta=0$ of $W$.

To reformulate the problem of Cauchy in $\left(\alpha, \beta, \phi, \theta_{1}, \cdots, \theta_{n-2}\right)$ -

- Compare M. H. Martin, loc. cit., p. 245. 
space we observe that the carrier $t=0$ becomes the hyperplane $\beta=-\alpha$ upon which, from (3), we assign

$$
u_{\phi}=u_{\phi}^{0}, \quad u_{\theta_{j}}=u_{\theta_{j}}^{0}, \quad u_{\alpha}=\left(u_{r}^{0}+u_{t}^{0}\right) / 2, \quad u_{\beta}=-\left(u_{r}^{0}-u_{t}^{0}\right) / 2
$$

as initial data. One would accordingly seek an expression for the value of the solution $u$ of $L(u)=0$, for $L(u)$ as defined in (4), along the edge $\alpha=\beta=\bar{t}$ of $W$ in terms of the above initial data carried by the face $\beta=-\alpha$ of $W$.

To solve the problem of Cauchy as originally formulated we apply the lemma of the preceding section to the closed surface $S_{n}$ which is the boundary of the wedge $W$ and obtain

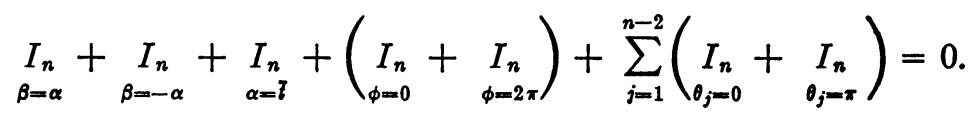

For single-valued solutions, $u$ must be periodic of period $2 \pi$ in $\phi$ and it follows from the definition of $\Phi$ that

$$
\underset{\phi=0}{I_{n}}+\underset{\phi=2 \pi}{I_{n}}=0
$$

since the external normals to $S_{n}$ have opposite directions on the faces $\phi=0, \phi=2 \pi$. Since $\Theta_{j}$ involves $f_{j}$, and $f_{j}$ contains $\sin \theta_{j}$ as a factor for $j=1, \cdots, n-2$, it is clear that

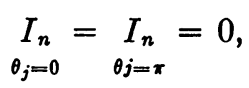

and the above result simplifies to

$$
\underset{\beta=\alpha}{I_{n}}+\underset{\beta=-\alpha}{I_{n}}+\underset{\alpha=I}{I_{n}}=0 .
$$

The integration of $I_{n}$ in (6) over $S_{n}$ yields

$$
\begin{aligned}
\int_{0}^{l} \int_{\omega_{n}}[-A+B]_{\beta=\alpha} f^{-1} d \omega_{n} d \alpha & -\int_{0}^{I} \int_{\omega_{n}}[A+B]_{\beta--\alpha} f^{-1} d \omega_{n} d \alpha \\
& \left.+\int_{-\downarrow}^{l} \int_{\omega_{n}} A\right]_{\alpha=l} f^{-1} d \omega_{n} d \beta=0
\end{aligned}
$$

and when we employ the definitions of $A$ and $B$, we find

$$
\begin{aligned}
& -\int_{0}^{l} \int_{\omega_{n}}\left[u_{\alpha} v_{\alpha}+u_{\beta} v_{\beta}\right]_{\beta=\alpha} d \omega_{n} d \alpha \\
& +\int_{0}^{l} \int_{\omega_{n}}\left[u_{\alpha} v_{\alpha}-u_{\beta} v_{\beta}\right]_{\beta=-\alpha} d \omega_{n} d \alpha+\int_{-l}^{l} \int_{\omega_{n}} u_{\beta} v_{\beta} \int_{\alpha=l} d \omega_{n} d \beta=0 .
\end{aligned}
$$


Up to this point $v$ has been any solution of the associate equation $M(v)=0$. For $v$ we now take the special solution ${ }^{6}$

$$
v=(\bar{t}-\alpha)^{(n-1) / 2(\bar{t}-\beta)^{(n-1) / 2},} \quad n \geqq 2 .
$$

This solution, termed the resolvent, is obtained by applying the ordinary method of separation of variables to $M(v)=0$ and plays the role of "Riemann's function." It is convenient to observe that

$\beta=\alpha$ implies $r=0, \alpha=t$,

$$
\frac{v_{\alpha}-v_{\beta}}{2}=0, \quad \frac{v_{\alpha}+v_{\beta}}{2}=-\frac{n-1}{2}(\bar{t}-t)^{n-2},
$$

$\beta=-\alpha$ implies $t=0, \alpha=r$,

$$
\begin{aligned}
& \frac{v_{\alpha}-v_{\beta}}{2}=-\frac{n-1}{2}\left(\bar{t}^{2}-r^{2}\right)^{(n-3) / 2} r, \\
& \frac{v_{\alpha}+v_{\beta}}{2}=-\frac{n-1}{2}\left(\bar{t}^{2}-r^{2}\right)^{(n-3) / 2} \bar{t},
\end{aligned}
$$

$\alpha=\bar{t}$ implies $v_{\beta}=0$.

More precisely, the last relations hold for $n \geqq 3$, and (8) holds as a result of integrating the fundamental identity (5) over the "wedge" $W$, all integrals involved being proper integrals. However, if $n=2$ then $v_{\beta}$ is infinite on $\alpha=\bar{t}$ and in order to obtain (8)-where improper integrals now appear-it is necessary to integrate first the identity (5) in $(\alpha, \beta, \phi)$-space over the smaller "wedge" $W_{\epsilon, \eta}$ whose cross section in the $\alpha \beta$-plane is bounded by the four straight lines

$$
\alpha=\beta, \alpha=-\beta, \beta=\bar{t}-\epsilon, \alpha=\bar{t}-\eta,
$$

where $0<\eta<\epsilon<\bar{t}$. Passing to the limit, letting $\eta \rightarrow 0$ first, and afterwards letting $\epsilon \rightarrow 0$, yields (8).

Thus the last term in (8) drops out altogether, eliminating the need for prescribed data on the characteristic half-cone, and the result is

$$
\begin{aligned}
\left.\int_{0}^{T} \int_{\omega_{n}}(\bar{t}-t)^{n-2} u_{t}\right|_{r=0} d \omega_{n} d t \\
\quad=\int_{0}^{T} \int_{\omega_{n}}\left[\left(\bar{t}^{2}-r^{2}\right)^{(n-3) / 2} \bar{t} u_{r}^{0}+\left(\bar{t}^{2}-r^{2}\right)^{(n-3) / 2} \cdot r \cdot u^{1}\right] d \omega_{n} d r
\end{aligned}
$$

where the integration on the left is performed on the axis of the cone

- Compare G. Darboux, loc. cit., p. 70, for $n=2$. 
C. Since

$$
\begin{aligned}
\int_{0}^{l} d t_{1} \int_{0}^{t_{1}} d t_{2} \cdots \int_{0}^{t_{m-2}} d t_{m-1}\left[\int_{0}^{t_{m-1}} f\left(t_{m}\right) d t_{m}\right] \\
=\int_{0}^{l} \frac{(\bar{t}-t)^{m-1}}{(m-1) !} f(t) d t,
\end{aligned}
$$

it follows that the preceding relation may be differentiated at least $n-1$ times with respect to $\bar{t}$. Differentiating $n-2$ times with respect to $\bar{t}$ yields the final formula:

$$
\begin{aligned}
& u\left(P_{\imath}\right)=u\left(P_{0}\right)+\frac{1}{(n-2) ! \omega_{n}} \\
& \cdot \frac{\partial^{n-2}}{\partial \bar{t}^{n-2}} \int_{0}^{I} \int_{\omega_{n}}\left[\left(\bar{t}^{2}-r^{2}\right)^{(n-3) / 2} \bar{t} u_{r}^{0}+\left(\bar{t}^{2}-r^{2}\right)^{(n-8) / 2} r u^{1}\right] d \omega_{n} d r .
\end{aligned}
$$

In the present notation, the usual formula ${ }^{7}$ for the solution of the Cauchy problem considered above may be written

$$
\begin{aligned}
u\left(P_{\imath}\right)= & \frac{1}{(n-2) ! \omega_{n}} \frac{\partial^{n-1}}{\partial \bar{t}^{n-1}} \int_{0}^{l} \int_{\omega_{n}}\left(\bar{t}^{2}-r^{2}\right)^{(n-3) / 2} \cdot r \cdot u^{0} d \omega_{n} d r \\
& +\frac{1}{(n-2) ! \omega_{n}} \frac{\partial^{n-2}}{\partial \bar{t}^{n-2}} \int_{0}^{l} \int_{\omega_{n}}\left(\bar{t}^{2}-r^{2}\right)^{(n-3) / 2} \cdot r \cdot u^{1} d \omega_{n} d r .
\end{aligned}
$$

The two formulas for $u\left(P_{\imath}\right)$ are easily seen to coincide, ${ }^{8}$ upon differentiating once with respect to $\bar{t}$ the first integral on the right-hand side of (10). This differentiation may be carried out directly under the integral sign if one first sets $r=\bar{t}_{\rho}$. A subsequent integration by parts then yields the result.

In conclusion, the above argument shows the uniqueness of the solution of Cauchy's problem. More precisely, if the Cauchy problem considered has a solution $u$ which possesses continuous second derivatives on $t>0$ and continuous first derivatives on $t \geqq 0$, then $u$ is given by formula (9).

\section{UNIVERSITY OF MARYLAND}

${ }^{7}$ R. Courant and D. Hilbert, Methoden der Mathematische Physik, vol. II, Berlin, 1937, p. 399.

${ }^{8}$ See M. H. Martin, loc. cit., page 244 , for the case $n=2$. 